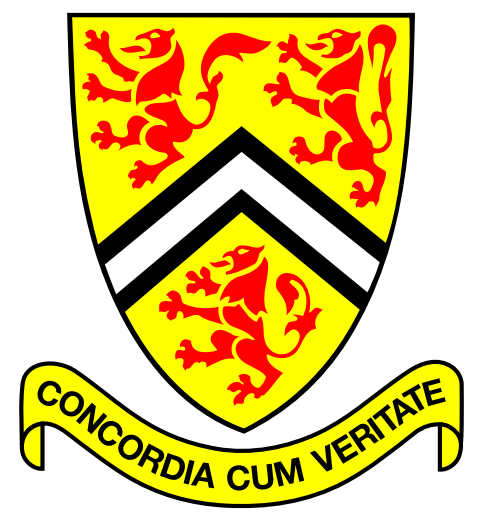

\title{
Turbo-Coded OFDM Transmission over a Nonlinear Channel
}

Hosein Nikopour(1), Amir K. Khandani(1), and S. Hamidreza Jamali(2)

(1) Coding \& Signal Transmission Laboratory

Department of Electrical \& Computer Engineering

University of Waterloo, Waterloo, ON N2L 3G1, Canada

(2) Department of Electrical \& Computer Engineering

University of Tehran, Tehran, Iran

Technical Report UW-E\&CE\#2004-16

June 2004 


\title{
Turbo-Coded OFDM Transmission over a Nonlinear Channel
}

\author{
Hosein Nikopour(1), Amir K. Khandani(1), and S. Hamidreza Jamali(2) \\ (1) Coding \& Signal Transmission Laboratory \\ Department of Electrical \& Computer Engineering \\ University of Waterloo, Waterloo, ON N2L 3G1, Canada \\ Tel: (519) 884-8552, Fax: (519) 888-4338 \\ (2) Department of Electrical \& Computer Engineering \\ University of Tehran, Tehran, Iran \\ e-mail: \{hossein, khandani\}@cst.uwaterloo.ca, hjamali@ut.ac.ir
}

\begin{abstract}
In this paper, deliberate level clipping and Turbo-coding are combined to achieve an Orthogonal Frequency Division Multiplexing (OFDM) transmission system with a low Peak-to-Average power Ratio (PAR) and a good performance. Using the linear approximation technique, we first modify the metric computation for the Turbo-decoding in order to consider the distortion effects of the nonlinearity, caused by the Cartesian clipping. The linear approximation of the nonlinear device is based on the Minimum Mean Square Error (MMSE) criterion. By exploiting the linear model, the receiver calculates a modified metric considering the effects of the nonlinearity. Also, this paper introduces a modified Turbo-decoder which simultaneously performs the data estimation and signal reconstruction. In other words, the Turbo-decoder iteratively recovers the clipped signal by using the estimated data, and then improves the data estimation by using the newly recovered signal. Numerical results are presented showing an improvement in the performance of the OFDM transmission system over the nonlinear channel, an increase in the efficiency of the High Power Amplifier (HPA), and/or an expansion of the transmitter coverage area.
\end{abstract}




\section{INTRODUCTION}

Orthogonal Frequency Division Multiplexing (OFDM), a popular type of multicarrier transmission [1]-[5], is an effective modulation technique for high-data-rate wireless and wire-line applications, including Digital Subscriber Line (DSL) [6], Digital Audio Broadcasting (DAB) [7], Digital Video Broadcasting (DVB) [8], and Wireless Local Area Network (WLAN) [9]-[11]. The main advantage of OFDM is its ability to encounter multipath fading without requiring complex equalizers [12]. Moreover, OFDM is a bandwidth efficient transmission system and can be easily implemented by the FFT [13].

The superposition of several subcarrier signals leads to a Gaussian-like time domain OFDM waveform [2], [3] with significant envelope variations or a high Peak-to-Average power Ratio (PAR) [14]. This undesirable feature renders the OFDM particularly sensitive to nonlinear distortions [15]-[19].

In practice, transmission devices such as the High Power Amplifier (HPA) have a limited dynamic range [20]. Therefore, to ensure a distortion-less transmission, hardware with a high power back-off is required, but it restricts the OFDM system from utilizing the transmitted power effectively.

There are many approaches to deal with this power control problem [21]-[38]. One class of approaches are based on generating OFDM signals with a low PAR [21]-[33]. Recently, many PAR reduction methods have been proposed in the literature. The most widely known techniques in this category are based on selective mapping [21]-[24], phase shifting [24]-[28], or some form of coding [29]-[33]. Selective mapping and phase shifting offer a considerable reduction of the PAR, but at the price of a significant increase of the overall system complexity. Coding techniques with the capabilities of both PAR reduction, as well as error correction (Reed-Muller codes) [29]-[31], are attractive. However, these codes significantly reduce the overall throughput of the system, especially if there is a relatively large number of subcarriers [29], [30].

Level clipping is a simple technique to reduce the PAR of the OFDM signal [34][38]. However, clipping is a nonlinear process, which distorts the transmitted signal [39]. 
OFDM signal can be clipped at the Nyquist rate or at over-sampling rates. Clipping the over-sampled signal produces in-band noise and out-of-band radiation, whereas clipping the Nyquist rate signal generates clipping noise that falls in-band resulting in a more serious degradation of the performance [34], [39]. Conventional coding techniques such as the Turbo-code [40]-[42] are attractive tools for mitigating such a high performance loss, caused by the nonlinear distortion [43], [44]. Consequently, the combination of clipping and Turbo-coding can be used as an effective approach for both PAR reduction and error correction with a reasonable coding redundancy and system complexity.

To mitigate the nonlinear distortion, a Turbo-decoder requires knowledge of the characteristic of the clipping noise. This paper shows that a linear approximation of the clipper in the Minimum Mean Square Error (MMSE) [45] sense can be used as an accurate model for a nonlinear device. After this model is employed for metric computation, the Turbo-decoder implicitly incorporates for the effects of the clipping distortion in the decoding procedure.

If the nonlinear characteristic of the transmitter is known, the nonlinear distortion is a deterministic function of the transmitted data. Therefore, by using the estimated data, after some initial Turbo-decoding iterations, the Turbo-decoder can partially compensate for the effects of the signal clipping. In other words, the Turbo-decoder recursively recovers the clipped signal by using the estimated data, and then uses the newly recovered signal to improve its data estimation.

The idea of the decision-aided reconstruction of the clipped signal in an uncoded OFDM system is introduced in [47], and further developed in [48]. In this paper, however, this idea is applied to a Turbo-coded OFDM system by adopting iterative decoding at the receiver end. Although, throughout this work, we assume that the clipping operation is performed in the baseband at the Nyquist sampling rate and the clipper is an ideal soft limiter, the suggested technique can be easily generalized to other forms of nonlinearities.

The rest of the paper is organized as follows. Section II is a brief description of the Turbo-coded OFDM transmitter under consideration. After the linear model of the clipper, 
based on the MMSE criterion, is derived in Section III, the linear model is adopted to modify the metric in Section IV. Section V introduces the corresponding Turbo-decoder and the process of the signal reconstruction. Section VI is devoted to some numerical examples. The paper finally concludes in Section VII.

Throughout this paper, $E\{\cdot\}$ denotes expectation, $j=\sqrt{-1}$, the small letters indicate the time domain samples, the capital letters represent the frequency domain samples, and the letters in bold denote the vectors of these time or frequency domain samples.

\section{OFDM OVERVIEW}

The baseband model of the underlying OFDM transmission system is represented in Fig. 1. Each input block of $k$ bits passes through a systematic Turbo-encoder [40] of rate $r_{c}(=k / \ell)$. The block of $\ell$ bits at the output of the Turbo-encoder is interleaved, and then mapped to $L(=\ell / m)$ complex samples, selected from $\mathcal{X}$, an $M$-ary Quadrature Amplitude Modulation ( $M$-QAM) constellation in which each point represents $m$ (= $\left.\log _{2} M\right)$ bits. This stream of $L$ symbols is partitioned into $L / N$ modulating vectors so that each has the dimension $N$. The final OFDM signal is the sum of the $N$ subcarriers, each being modulated by the corresponding element of the modulating vector.

If $X_{i}, i=0,1, \ldots, N-1$, is defined as the QAM symbol, associated with the $i$ th element of the modulating vector $\mathbf{X}$ then the vector of the Nyquist rate samples of the OFDM baseband signal is expressed as

$$
\begin{aligned}
\mathbf{x} & =\left(x_{1}, x_{2}, \ldots, x_{N-1}\right) \\
& =\operatorname{IDFT}(\mathbf{X}),
\end{aligned}
$$

where

$$
x_{n}=\frac{1}{\sqrt{N}} \sum_{i=0}^{N-1} X_{i} e^{j \frac{2 \pi i n}{N}}, \quad n=0,1, \ldots, N-1 .
$$

We assume that the average power of the QAM symbols is equal to $2 \sigma^{2}$. Due to the orthogonality of the subcarriers, the average power of the OFDM samples is also the same and is equally distributed among the real and imaginary parts. 


\section{NONLINEARITY MODELING}

The clipper is the source of the nonlinearity of the model in Fig. 1. Consequently, this section first describes the characteristics of the nonlinear device, and then models the distortion effects of the nonlinearity on the OFDM signal.

Typically, we can distinguish between two classes of nonlinearity [49]: 1) Cartesian distortion that acts separately on the real part and the imaginary part of the baseband signal (e.g. in D/A conversion), and 2) polar distortion that acts on the envelope and phase of the OFDM signal (e.g. in high power amplification). According to Fig. 2, the Cartesian distortion limits the signal within a square, whereas the polar distortion limits the signal in a circle. In this paper, without the loss of generality, we confine our study to the Nyquist rate Cartesian soft limiter, which belongs to the first class.

Most of the common nonlinear devices in the transmitter can be accurately modeled as memoryless systems [48]. The distorted signal at the output of the memoryless clipper is written as

$$
d=g(x)=\left\{\begin{array}{lr}
-A, & x \leq-A, \\
x, & -A<x<A, \\
A, & x \geq A,
\end{array}\right.
$$

where $A$ is the saturation level. With some misuse of the notation, $x$ denotes either the real part or the imaginary part of $x_{n}$. If $d$ is rewritten as follows:

$$
d=x+c,
$$

then $c$ represents the distortion term of the clipped signal.

We know that when $N$ is a large number, $x$ can be modeled as a Gaussian random variable [2], [3] with the Probability Distribution Function (PDF), given by

$$
p_{x}(x)=\frac{1}{\sigma} G(x / \sigma)
$$

where $G(\tau)=\frac{1}{\sqrt{2 \pi}} e^{-\tau^{2} / 2}$ is the zero-mean, unit-variance Gaussian function. As a result, 
the average power of the clipped OFDM sample can be obtained as follows:

$$
\begin{aligned}
\sigma_{d}^{2}= & \int_{-\infty}^{\infty} g(x) p_{x}(x) d x \\
= & \sigma^{2}\left[1-2 Q(\sqrt{i b o})-\sqrt{2 i b o / \pi} e^{-i b o / 2}\right. \\
& +2 i b o Q(\sqrt{i b o})]
\end{aligned}
$$

where $Q(x)=\int_{x}^{\infty} G(\tau) d \tau$. The input back-off, ibo, is defined as the ratio of the maximum allowable input power to the average input power (e.g., refer to [10]), which is described as

$$
i b o=A^{2} / \sigma^{2}
$$

The output back-off, obo, is another parameter of the clipper which indicates the relative level of the clipping, and is defined as the ratio of the maximum output power to the average power of the clipped signal, i.e.,

$$
o b o=A^{2} / \sigma_{d}^{2}
$$

Indeed, the parameter obo is equal to the maximum PAR of the clipped signal. If (6) is substituted into (8), obo is described as a function of $i b o$,

$$
\begin{aligned}
\text { obo }= & i b o /\left[1-2 Q(\sqrt{i b o})-\sqrt{2 i b o / \pi} e^{-i b o / 2}\right. \\
& +2 i b o Q(\sqrt{i b o})]
\end{aligned}
$$

which is illustrated in Fig. 3. We can see that for the high values of back-off, obo and $i b o$ are approximately the same.

Let us approximate the nonlinear function $g(x)$ by the linear function $\alpha x$. The linear coefficient $\alpha$ can be set so that the error of the approximation,

$$
e=g(x)-\alpha x
$$

is minimized in the mean square sense, i.e.,

$$
\alpha=\arg \min _{\alpha} E\left\{e^{2}\right\}
$$


As a result,

$$
\alpha=1-2 Q(\sqrt{i b o})
$$

and

$$
\begin{aligned}
\sigma_{e}^{2}= & E\left\{e^{2}\right\}=\sigma_{d}^{2}-\alpha^{2} \sigma^{2} \\
= & \sigma^{2}[2 Q(\sqrt{i b o})(1-2 Q(\sqrt{i b o})) \\
& \left.-\sqrt{2 i b o / \pi} e^{-i b o / 2}+2 i b o Q(\sqrt{i b o})\right] .
\end{aligned}
$$

Consequently, according to the following equation:

$$
d=\alpha x+e
$$

the effect of the nonlinear distortion is modeled as an attenuation $\alpha$ and an additive "nonlinear" noise $e$. In addition, due to the property of the MMSE approximation, the nonlinear noise is uncorrelated with the input signal $x$.

According to (14), the signal to nonlinear noise power ratio is defined as

$$
S N R_{e}=\frac{\alpha^{2} \sigma^{2}}{\sigma_{e}^{2}} .
$$

If (12) and (13) are substituted into (15), $S N R_{e}$ is expressed as a function of $i b o$ as follows:

$$
\begin{aligned}
S N R_{e}= & (1-2 Q(\sqrt{i b o}))^{2} / \\
& {[2 Q(\sqrt{i b o})(1-2 Q(\sqrt{i b o}))} \\
& \left.-\sqrt{2 i b o \pi} e^{-i b o / 2}+2 i b o Q(\sqrt{i b o})\right] .
\end{aligned}
$$

Fig. 4 exhibits that $S N R_{e}$ is obviously an ascending function of the $i b o$, because increasing $i b o$ reduces the distortion effects of the clipper.

Although these equations are derived for the clipper, their generalization to other forms of $g(x)$ is straightforward. 


\section{OFDM RECEIVER}

The transmitted signal $d_{n}$ is corrupted by an Additive White Gaussian Noise (AWGN) $w_{n}$ with an average power equal to $2 \sigma_{w}^{2}$. Therefore, the transmit signal to the AWGN power ratio is equal to

$$
S N R_{w}=\frac{\sigma_{d}^{2}}{\sigma_{w}^{2}}
$$

The received signal,

$$
r_{n}=d_{n}+w_{n}
$$

passes through the OFDM FFT demodulator where the output is expressed as

$$
R_{i}=D_{i}+W_{i}
$$

The Maximum A-posteriori Probability (MAP) [45], [46] receiver requires the knowledge of the following conditional probability:

$$
P\left(X_{i}=X \mid R_{0}, R_{1}, \ldots, R_{N-1}\right)
$$

to compute the metrics that are used in the Turbo-decoding. The presence of the nonlinear device results in a dependency among the subcarriers. An ideal Turbo-decoder uses (20) to compute the Turbo-decoding metric. However, due to the enormous computational complexity that is associated with (20), we employ the following conditional probability:

$$
P\left(X_{i}=X \mid R_{i}\right)
$$

If it is assumed that all constellation points are acquired with equal probability, the following conditional distribution probability is used to compute the Turbo-decoding metric:

$$
p\left(R_{i} \mid X_{i}=X\right)
$$

Using the linear model, we rewrite the received signal as

$$
R_{i}=\alpha X_{i}+E_{i}+W_{i}
$$


where $E_{i}, i=0,1, \ldots, N-1$, is a zero-mean, nonlinear noise in the frequency domain that is described as

$$
E_{i}=\frac{1}{\sqrt{N}} \sum_{n=0}^{N-1} e_{n} e^{-j \frac{2 \pi i n}{N}}
$$

or

$$
\mathbf{E}=\operatorname{DFT}(\mathbf{e})
$$

Since $N$ is large, by invoking the Central Limit theorem, $E_{i}$ can be modeled as a Gaussian variable [15], [18], [49]. This is accurate, especially in a practical situation, where the clipping level $A$ is not very large, causing most of the $e_{n}$ samples to have nonzero values. Consequently, $E_{i}$ is, approximately, a Gaussian variable, independent of $X_{i}$ and with a power that is equal to $2 \sigma_{e}^{2}$. Considering this fact and according to (23), we represent the Turbo-decoder metric as

$$
p\left(R_{i} \mid X_{i}=X\right) \propto \exp \left(-\left|R_{i}-\alpha X\right|^{2} / 2 \sigma_{t}^{2}\right)
$$

where

$$
2 \sigma_{t}^{2}=2 \sigma_{e}^{2}+2 \sigma_{w}^{2}
$$

is the total noise power.

Without using the linear model, it is directly shown in the appendix that for a large $N$,

$$
E\left\{R_{i} \mid X_{i}=X\right\} \approx \alpha X
$$

This is a match to (23) and (26), and therefore, justifies the validity of the linear model.

According to (23), the signal to the total noise power ratio is

$$
S N R_{t}=\frac{\alpha^{2} \sigma^{2}}{\sigma_{t}^{2}},
$$

which, by using (15), (17), and (27), is

$$
S N R_{t}=\frac{S N R_{e} S N R_{w}}{1+S N R_{e}+S N R_{w}} .
$$




\section{ModificAtion of THE Turbo-Decoder}

The conventional Turbo-decoder cannot mitigate the effects of the nonlinear distortion. Therefore, to achieve a reasonable performance through a nonlinear transmission, the Turbo-decoder must be modified to correspond to the behavior of the channel. The block of the metric calculation in Fig. 1 uses $p\left(R_{i} \mid X_{i}=X\right)$ to compute the probability of the $p$ th bit $p=0,1, \ldots, m-1$, associated to the received signal $R_{i}$ as follows:

$$
P\left(b_{p}=b ; R_{i}, i\right) \propto \sum_{X \in \mathcal{X}(p, b)} p\left(R_{i} \mid X_{i}=X\right) .
$$

In (31), $b \in\{0,1\}$ and $\mathcal{X}(p, b) \subset \mathcal{X}$ is the set of the QAM points for which the $p$ th bit is equal to $b$.

The Logarithmic Likelihood Ratio (LLR) is related to the bit probabilities as follows [40]:

$$
L_{L} R_{p}\left(R_{i}, i\right)=10 \log \frac{P\left(b_{p}=1 ; R_{i}, i\right)}{P\left(b_{p}=0 ; R_{i}, i\right)} .
$$

The modified Turbo-decoder uses (26) to incorporate the distortion, caused by the nonlinearity. Substituting (26) in (32), we can write

$$
L L R_{p}\left(R_{i}, i\right)=10 \log \frac{\sum_{X \in \mathcal{X}(p, 1)} \exp \left(-\left|R_{i}-\alpha X\right|^{2} / \sigma_{t}^{2}\right)}{\sum_{X \in \mathcal{X}(p, 0)} \exp \left(-\left|R_{i}-\alpha X\right|^{2} / \sigma_{t}^{2}\right)} .
$$

Therefore, the Turbo-decoder requires the knowledge of the clipping parameters to calculate the modified metric.

As described in the standards [6]-[11], the pilots and data subcarriers are transmitted simultaneously in the same OFDM symbol. Therefore, the pilots like the data subcarriers are affected by the nonlinear device. Fortunately, in this situation, the clipper parameters can be found by using the pilots during the channel and noise power estimation operations [50] without an additional estimator block. In this way, a conventional Turbo-decoder implicitly calculates the modified metric.

The sequence of the modified LLRs, corresponding to all $\ell$ coded bits after channel deinterleaving, passes through the Turbo-decoder to estimate $k$ information bits. 
This procedure assumes that the receiver has no knowledge of the distortion term and can only model the nonlinearity effects as signal attenuation and additive noise. The performance of the Turbo-decoder can be further enhanced by estimating the distortion term toward compensating the effect of the nonlinear device. According to (4), the received signal over the $i$ th subcarrier can be written as

$$
R_{i}=X_{i}+C_{i}+W_{i}
$$

Obviously, $\mathbf{C}=\left(C_{0}, C_{1}, \ldots, C_{N-1}\right)$ is a function of the transmitted QAM vector, $\mathbf{X}$

$$
\mathbf{C}=\operatorname{DFT}(\mathbf{g}(\operatorname{IDFT}(\mathbf{X})))-\mathbf{X}
$$

where $\mathbf{g}(\mathbf{Y})=\left(g\left(y_{1}\right), g\left(y_{2}\right), \ldots, g\left(y_{N-1}\right)\right)$.

Since $\mathbf{X}$ is unknown, the receiver must use an estimate of $\mathbf{C}$, denoted as $\hat{\mathbf{C}}$, by knowing $g(\cdot)$ and having an estimate of $\mathbf{X}$ from the previous iterations of the Turbo-decoding. This process can be continued in an iterative manner.

Let us assume $P_{t}(b), t=0,1, \ldots, \ell-1, b \in\{0,1\}$ is the interleaved output of the Turbo-decoder which denotes the probability of the $t$ th transmitted bit to be equal to $b$. Since the interleaved bits are approximately independent, the $i$ th transmitted QAM symbol, $i=0,1, \ldots, L-1$, is $X=\left\{b_{0}, b_{1}, \ldots, b_{m-1} \mid b_{p}=0\right.$ or $\left.1,0 \leq p \leq m-1\right\} \in \mathcal{X}$ with the probability $P_{i}(X)$ being expressed as

$$
\begin{aligned}
P_{i}(X) & =P_{i}\left(\left\{b_{0}, b_{1}, \ldots, b_{m-1}\right\}\right) \\
& \approx \prod_{p=0}^{m-1} P_{i m+p}\left(b_{p}\right) .
\end{aligned}
$$

The estimate of $X_{i}$ is defined as the average of the constellation points, i.e.,

$$
\hat{X}_{i}=\sum_{X \in \mathcal{X}} P_{i}(X) X
$$

Let us define $\nabla .(\cdot)$ as the previous procedure that estimates $X_{i}$ 's of the all transmitted OFDM symbols from the sequence of $L L R_{p}\left(R_{i}, i\right), p=0,1, \ldots, m-1, i=0,1, \ldots, L-$ 1, denoted as LLR, i.e.,

$$
\left(\hat{X}_{0}, \hat{X}_{1}, \hat{X}_{2}, \ldots\right)=\nabla_{I}(\mathbf{L L R})
$$


where $I$ is the number of Turbo-decoding iterations. The modified Turbo-decoding procedure can be summarized in the following steps, starting with $u=1$. In the following discussion, the subscript within the parentheses denotes the stage number.

1) The LLR of the first stage $\mathbf{L L R}^{(0)}$ is calculated as a function of the received signal by utilizing (33).

2) The $u$ th estimate of $X_{i}$ 's, after the $(u-1)$ th reconstruction of the received signal, is $(\operatorname{see}(38))$

$$
\left(\hat{X}_{0}^{(u)}, \hat{X}_{1}^{(u)}, \hat{X}_{2}^{(u)}, \ldots\right)=\nabla_{I}\left(\mathbf{L} \mathbf{L} \mathbf{R}^{(u-1)}\right)
$$

3) According to (35), the estimate of the distortion term is

$$
\hat{\mathbf{C}}^{(u)}=\operatorname{DFT}\left(\mathbf{g}\left(\operatorname{IDFT}\left(\hat{\mathbf{X}}^{(u)}\right)\right)\right)-\hat{\mathbf{X}}^{(u)} .
$$

This procedure is performed on all the OFDM symbols.

4) The received signal is reconstructed as follows:

$$
R_{i}^{(u)}=R_{i}-\hat{C}_{i}^{(u)}
$$

5) If we neglect the remaining distortion term after the signal reconstruction, then, according to (34) and (41), the new LLR can be written as

$$
\begin{array}{r}
L L R_{p}^{(u)}\left(R_{i}^{(u)}, i\right)= \\
10 \log \frac{\sum_{X \in \mathcal{X}(p, 1)} \exp \left(-\left|R_{i}^{(u)}-X\right|^{2} / 2 \sigma_{w}^{2}\right)}{\sum_{X \in \mathcal{X}(p, 0)} \exp \left(-\left|R_{i}^{(u)}-X\right|^{2} / 2 \sigma_{w}^{2}\right)} .
\end{array}
$$

6) The next stage of the signal reconstruction begins with $u \leftarrow u+1$. Go to the step 2 .

If $U$ represents the number of times that the Turbo-decoder with $I$ iterations is used, then the total number of the Turbo-decoding iterations is $H=I U$. Therefore, this modified decoding algorithm is comparable with the classical Turbo-decoder with a total of $H$ iterations. However, this algorithm requires two additional FFTs and one additional LLR computation in the decoder for each stage of the signal reconstruction. 


\section{NUMERICAL RESULTS}

In all of the following, the OFDM system has a Turbo-code with a rate of $\frac{1}{2}$, a memory size of 4 , and an interleaver length of 4096, and therefore, the channel interleaver is the length, 8192 bits.

Fig. 5 displays the performance of the 256-QAM OFDM system for the different subcarrier numbers. The vertical axis is the Bit Error Rate (BER), and the horizontal axis is the signal to noise ratio per information bit that is defined as

$$
\frac{E_{b}}{N_{o}}=\frac{S N R_{w}}{r_{c} m}
$$

The Turbo-decoder has 12 iterations without any signal reconstruction, and the Turbodecoder uses only the modified metric. From this point in the paper, this type of Turbodecoder is called the "classical decoder". In Fig. 5, ibo is set to $5 \mathrm{~dB}$. The accuracy of the modified metric depends on the number of subcarriers. As plotted in Fig. 5, the performance of the 1024-subcarrier system is better than the system with $N=64$ and $N=256$. Although the metric modification significantly improves the performance, but there is still a substantial performance degradation, compared to the performance of the system in an ideal channel.

The results of exploiting the proposed Turbo-decoder (the modified decoder) are illustrated in Fig. 6. The system achieves a better performance, if the number of the signal reconstruction iterations is increased. It seems that $U$ is a dominant factor in comparison to $I$. For example, a system with $I=2$ and $U=4(H=8)$ has approximately the same performance as the system with $I=3$ and $U=4(H=12)$. However, for $H=12$, it is possible to increase $U$ to 6 which leads to a further improvement. As Fig. 6 confirms, there is a little improvement for $U>6$; consequently, the performance of the system with $I=3$ and $U=8(H=24)$ appears to be the best that can be achieved by this modified Turbo-decoder. In this case, the degradation in performance, due to the signal clipping, is approximately $1.4 \mathrm{~dB}$ when the BER is equal to $10^{-4}$.

In Fig. 7, we decrease the number of the subcarriers from 256 to 64 . The proposed decoder still converges so that the performance is improved but at a slower rate. 
The results in Fig. 8 are for $N=256, M=256$, and a higher clipping level of $i b o=7 \mathrm{~dB}$. Obviously, due to less clipping distortion, the remaining degradation, with respect to the ideal channel, is less than $0.8 \mathrm{~dB}$ for $I=2$ and $U=6(H=12)$.

Fig. 9 depicts the BER of the 1024-subcarrier, 256-QAM OFDM system for practical conditions as a function of the amplifier clipping level, obo. These results are for several values of the noise back-off $n b o$, which is defined as follows:

$$
n b o=\frac{A^{2}}{\sigma_{w}^{2}} .
$$

In fact, $n b o$ is the maximum achievable signal to noise power ratio in a transmission channel for which the maximum transmit power is $2 A^{2}$, and the noise power of the channel is $2 \sigma_{w}^{2}$. Consequently, $n b o$ indicates the quality of the transmission channel. When the channel is in a deep fade (or equivalently the relative noise power is high), $n b o$ is low; in contrast, when the received power is high, the channel is in a good condition, and $n b o$ has a relatively high value.

Fig. 9 exhibits that for a fixed $n b o$, when $o b o$ is high, and as a result, the HPA works in the linear region, by decreasing obo (or equivalently by increasing the average transmit power), the system performance improves. However, this situation changes when the HPA approaches the saturation level, and the nonlinear noise becomes dominant. Therefore, after a specific obo, which is the optimum operating point of the HPA, despite an increase in the transmit power, the nonlinear noise distorts the transmit signal and prevents the system from achieving a better performance. Consequently, the HPA is not able to effectively utilize all of its dynamic range due to the nonlinearity. This causes the system to fail, especially when the receiver is far from the transmitter, or when the channel is in deep fade and so, $n b o$ is relatively low. For example, for $n b o=22.5 \mathrm{~dB}$, the classical decoder is not able to achieve a BER of less than $1.27 \times 10^{-2}$.

Fig. 9 compares the performance of the classical Turbo-decoder, $I=12, U=1(H=$ $12)$, and the modified Turbo-decoder, $I=3, U=4(H=12)$. When the channel has a good quality; for example $n b o=25 \mathrm{~dB}$, both decoders behave approximately the same, because the system can achieve a good performance with a relatively low transmit 
power in which obo is sufficiently high, and the HPA behaves like a linear system. However, if the channel quality degrades; for example $n b o$ drops to $23 \mathrm{~dB}$, then the transmitter has to increase the transmit power to keep the system performance within a reasonable range at an acceptable level. In this situation, the classical decoder cannot offer a performance that is better than $1.3 \times 10^{-3}$, whereas the best BER of the modified decoder is approximately $1.88 \times 10^{-4}$. Therefore, the ability of the modified decoder, to improve the system performance, is especially pronounced in a degraded channel condition (when the transmit power is required to be high, and the HPA is forced to operate in the nonlinear region with a comparatively low obo).

Fig. 10 illustrates the BER of the classical and the modified decoder as a function of $n b o$ and for $o b o=6.5,7.0$, and 7.5 dB. These results are extracted from Fig. 9. Fig. 10 depicts that the modified decoder can handle the worse channel quality while holding the BER at the same level as the BER of the classical decoder, operating in a better channel condition. For example, the gain of $n b o$ is approximately $0.6 \mathrm{~dB}$ for BER $=10^{-5}$ and $o b o=7.0 \mathrm{~dB}$, indicating that for the modified decoder, the signal attenuation can be $0.6 \mathrm{~dB}$ greater, in comparison to that of the classical decoder, while maintaining a BER that is equal to $10^{-5}$. Therefore, by exploiting the modified decoder, the transmitter can either expand its coverage area without requiring additional power, or retain its coverage area, and the average output power is decreased by $0.6 \mathrm{~dB}$.

The average transmit power gain, with the value of the BER as a parameter, is offered in Fig. 11, in which the classical and modified decoder are compared. These BERs are selected at the optimum performance of the classical decoder so that the dark points correspond to the optimum operating point of the HPA for a given nbo. According to this figure, the modified decoder can achieve the same performance as the classical one but with a lower transmit power (higher obo). This is the case for $o b o$ at approximately the optimum point of operation, where obo is relatively low, and the HPA operates near the nonlinear region. Therefore, by using the modified decoder, the transmission system can save the power through the higher obo selection. 
The behavior of the classical and the modified decoder at the optimum operating points are compared in Fig. 12(a) and (b). The minimum BER is shown as a function of $n b o$ in Fig. 12(a). Similar to Fig. 10, this figure signifies that the functionality threshold of the modified decoder is approximately $0.5 \mathrm{~dB}$ higher than the functionality threshold of the classical one. Fig. 12(b) illustrates the minimum BER as a function of the optimum obo. Each point in each curve indicates the optimum operation point of the amplifier. As shown in Fig. 12(b), the optimum obo of the modified decoder is approximately $0.4 \mathrm{~dB}$ less than the optimum point of the classical decoder. This demonstrates that the modified decoder utilizes the dynamic range of the HPA more effectively; i.e., the HPA efficiency, in converting the DC to AC power, is better when the system performs at the optimum operating point. It is clear that with the same BER, the channel quality of the modified receiver is worse than that of the classical one.

\section{CONCLUSION}

The combination of clipping and Turbo-coding is used to reduce the PAR, and simultaneously, achieve an acceptable performance with a reasonable redundancy. First, we demonstrate that the linear MMSE approximation of the memoryless, nonlinear device leads to an almost optimal symbol-wise metric. This linear model is more accurate for a large number of subcarriers. If the Turbo-decoder metric is computed with this model, the Turbo-decoder can reduce the BER by several orders of magnitude. In the next step, the Turbo-decoder is modified to recursively reconstruct the distorted signal during the decoding iterations. The numerical examples confirm that for a fixed number of decoding iterations, the modified Turbo-decoder results in a noticeable improvement in the BER with a slight increase in the complexity. Finally, the modified decoder is tested in a practical situation. The simulation results prove that a system, in which the modified decoder is exploited, can develop its coverage area and use the dynamic range of the HPA more effectively. 


\section{APPENDIX}

PROOF OF (28)

Let us start with $E\left\{d_{n} \mid X_{i}=X\right\}, n, i=0,1, \ldots, N-1$. According to (2), $x_{n}$, $n=0,1, \ldots, N-1$, can be rewritten as follows:

$$
x_{n}=\bar{x}_{n}^{(i)}+\frac{1}{\sqrt{N}} \sum_{q=0}^{N-1} X_{q} e^{j \frac{2 \pi q n}{N}},
$$

where

$$
\bar{x}_{n}^{(i)}=\frac{1}{\sqrt{N}} X e^{j \frac{2 \pi i n}{N}}
$$

is the mean of $x_{n} \mid X_{i}=X$. When $N$ is large, the summation term in (45) is, approximately, a complex Gaussian process with a variance that is expressed as follows:

$$
2 \tilde{\sigma}^{2}=\frac{N-1}{N} 2 \sigma^{2}
$$

Therefore, if $x$ and $\bar{x}$ respectively denote either the real part or the imaginary part of $x_{n}$ and $\bar{x}_{n}^{(i)}$, then the PDF of $x \mid X_{i}=X$ can be expressed as follows:

$$
p_{x \mid X}\left(x \mid X_{i}=X\right)=\frac{1}{\tilde{\sigma}} G\left(\frac{x-\bar{x}}{\tilde{\sigma}}\right) .
$$

According to (3), the clipped signal is a function of $x$, and then, we can easily show that

$$
E\left\{d \mid X_{i}=X\right\}=\int_{-\infty}^{\infty} g(x) p_{x \mid X}\left(x \mid X_{i}=X\right) d x
$$

If (3) and (48) are substituted into (49), after some manipulations, we can write

$$
\begin{aligned}
E\left\{d \mid X_{i}=X\right\}= & \sigma\left\{\left[G\left(\xi^{+}\right)-G\left(\xi^{-}\right)\right]\right. \\
& \left.-\left[\xi^{+} Q\left(\xi^{+}\right)-\xi^{-} Q\left(\xi^{-}\right)\right]\right\}+\bar{x},
\end{aligned}
$$

where

$$
\xi^{ \pm}=\frac{A \pm \bar{x}}{\tilde{\sigma}}
$$

If $N \rightarrow \infty$, it is clear from (46), (47), (51), and (7) that

$$
\left\{\begin{array} { l } 
{ \overline { x } \longrightarrow 0 } \\
{ \tilde { \sigma } ^ { 2 } \longrightarrow \sigma ^ { 2 } }
\end{array} \Longrightarrow \left\{\begin{array}{l}
\xi^{ \pm} \longrightarrow \sqrt{i b o} \\
\xi^{+}-\xi^{-} \longrightarrow \frac{2 \bar{x}}{\sigma},
\end{array}\right.\right.
$$


and then,

$$
\left\{\begin{array}{l}
G\left(\xi^{+}\right)-G\left(\xi^{-}\right) \longrightarrow 0, \\
\xi^{+} Q\left(\xi^{+}\right)-\xi^{-} Q\left(\xi^{-}\right) \longrightarrow \frac{2 \bar{x}}{\sigma} Q(\sqrt{i b o}) .
\end{array}\right.
$$

Therefore, according to (53) and (12), when $N$ is large, (50) reduces to

$$
E\left\{d_{n} \mid X_{i}=X\right\} \approx \alpha \bar{x}_{n}^{(i)}
$$

Transforming from the time to the frequency domain, (54) is changed as follows:

$$
E\left\{D_{h} \mid X_{i}=X\right\} \approx \frac{1}{\sqrt{N}} \sum_{n=0}^{N-1} \alpha \bar{x}_{n}^{(i)} e^{-j \frac{2 \pi h n}{N}}, \quad h=0,1, \ldots, N-1,
$$

which, by using (46), is reduced to

$$
E\left\{D_{h} \mid X_{i}=X\right\} \approx \alpha X \delta_{h-i}
$$

where $\delta_{\mu}$ is a Dirac function. Therefore

$$
E\left\{D_{i} \mid X_{i}=X\right\} \approx \alpha X .
$$

Since $W_{i}$ is a zero mean noise, (28) can be inferred from (19) and (57).

\section{REFERENCES}

[1] J. Bingham, "Multicarrier modulation for data transmission: an idea whose time has come," IEEE Commun. Mag., vol. 28, pp. 5-14, May 1990.

[2] W. Y. Zou and Y. Wu, “COFDM: an overview," IEEE Trans. Broadcast., vol. 41, pp. 1-8, Mar. 1995.

[3] L. Litwin, "An introduction to multicarrier modulation," IEEE Potentials, vol. 19, pp. 36-38, Apr.May 2000.

[4] Z.Wang and G. B. Giannakis, "Wireless multicarrier communications," IEEE Signal Processing Mag., pp. 29-48, May 2000.

[5] R. van Nee and R. Prasad, OFDM Wireless Multimedia Communications. London, UK: Artech House, 2000.

[6] Asymmetrical Digital Subscriber Line (ADSL) Transceivers, ITU-T Recommendation G.992.1, July 1999. 
[7] Radio Broadcast Systems Digital Audio Broadcasting (DAB) to Mobile, Portable, and Fixed Receivers, Eur. Telecommun. Standards Inst. (ETSI), ETS 300 401, May 1997.

[8] Digital Video Broadcasting (DVB-T); Framing Structure, Channel Coding, and Modulation for Digital Terrestrial Television, Eur. Telecommun. Standards Inst. (ETSI), ETS 300 744, Dec. 2001.

[9] Broadband Radio Access Networks (BRAN); HIPERLAN Type 2; Physical (PHY) Layer, Eur. Telecommun. Standards Inst. (ETSI), RTS/BRAN-0023003-R2, Feb. 2001.

[10] Supplement to Standard for Telecommunications and Information Exchange Between Systems LAN/MAN Specific Requirements - Part 11: Wireless LAN Medium Access Control (MAC) and Physical Layer (PHY) Specifications: High Speed Physical Layer in the 5 GHz Band, IEEE 802.11a, July 1999.

[11] R. van Nee, G. Awater, M. Morikura, H. Takahashi, M. Webster, and K. W. Halford, "New high-rate wireless LAN standards," IEEE Commun. Mag., vol. 37, pp. 82-88, Dec. 1999.

[12] L. J. Cimini, Jr., "Analysis and simulation of a digital mobile channel using orthogonal frequency division multiplexing," IEEE Trans. Commun., vol. 33, pp. 665-675, July 1985.

[13] S. B. Weinstein and P. M. Ebert, "Data transmission by frequency-division multiplexing using the discrete Fourier transform,” IEEE Trans. Commun. Technol., vol. 19, pp. 628-634, Oct. 1971.

[14] H. Ochiai and H. Imai, "On the distribution of the peak-to-average power ratio in OFDM signals," IEEE Trans. Commun., vol. 49, pp. 282-289, Feb. 2001.

[15] Q. Shi, "OFDM in bandpass nonlinearity," IEEE Trans. Consumer Electron., vol. 42, pp. 253-258, Aug. 1996.

[16] C.-L. Liu, "The effect of nonlinearity on a QPSK-OFDM-QAM signal," IEEE Trans. Consumer Electron., vol. 43, pp. 443-447, Aug. 1997.

[17] S. Merchan, A. G. Armada, and J. L. Garcia, "OFDM performance in amplifier nonlinearity," IEEE Trans. Broadcast., vol. 44, pp. 106-114, Mar. 1998.

[18] P. Banelli, G. Baruffa, and S. Cacopardi, "Effects of HPA non linearity on frequency multiplexed OFDM signals," IEEE Trans. Broadcast., vol. 47, pp. 123-136, June 2001.

[19] C. van den Bos, M. H. L. Kouwenhoven, and W. A. Serdijn, "Effect of smooth nonlinear distortion on OFDM symbol error rate," IEEE Trans. Commun., vol. 49, pp. 1510-1514, Sept. 2001.

[20] A. Barbieri, G. Cariolaro, and L. Vangelista, "Nonlinear models of TWT revisited for OFDM systems," in Proc. of the 38th Midwest Symposium on Circuits and Systems, vol. 1, Aug. 1996, pp. 522-525.

[21] P. V. Eetvelt, G. Wade, and M. Tomlinson, "Peak to average power reduction for OFDM schemes by selective scrambling," Electronics Letters, vol. 32, pp. 1963-1964, Oct. 1996. 
[22] R. W. Buml, R. F. H. Fischer, and J. B. Huber, "Reducing the peak-to average power ratio of multicarrier modulation by selected mapping," Electronics Letters, vol. 32, pp. 2056-2057, Oct. 1996.

[23] H. Breiling, S. Muller-Weinfurtner, and J. Huber, "SLM peak-power reduction without explicit side information," IEEE Commun. Lett., vol. 5, pp. 239-241, June 2001.

[24] K. Sathananthan and C. Tellambura, "Partial transmit sequence arid selected mapping schemes to reduce ICI in OFDM systems," IEEE Commun. Lett., vol. 6, pp. 313-315, Aug. 2002.

[25] L. J. Cimini, Jr. and N. R. Sollenberger, "Peak-to-average power ratio reduction of an OFDM signal using partial transmit sequences," IEEE Commun. Lett., vol. 4, pp. 86-88, Mar. 2000.

[26] S. G. Kang, J. G. Kim, and E. K. Joo, "A novel subblock partition scheme for partial transmit sequence OFDM," IEEE Trans. Broadcast., vol. 45, pp. 333-338, Sept. 1999.

[27] W. S. Ho, A. Madhukumar, and F. Chin, "Peak-to-average power reduction using partial transmit sequences: a suboptimal approach based on dual layered phase sequencing," IEEE Trans. Broadcast., vol. 49, pp. 225-231, June 2003.

[28] O.-J. Kwon and Y.-H. Ha, "Multi-carrier PAP reduction method using sub-optimal PTS with threshold," IEEE Trans. Broadcast., vol. 49, pp. 232-236, June 2003.

[29] J. Davis and J. Jedwab, "Peak-to-mean power control in OFDM, Golay complementary sequences, and Reed-Muller codes," IEEE Trans. Inform. Theory, vol. 45, pp. 2397-2417, Nov. 1999.

[30] K. Paterson, "Generalized Reed-Muller codes and power control in OFDM modulation," IEEE Trans. Inform. Theory, vol. 46, pp. 104-120, Jan. 2000.

[31] K. Paterson and A. Jones, "Efficient decoding algorithms for generalized Reed-Muller codes," IEEE Trans. Commun., vol. 48, pp. 1272-1285, Aug. 2000.

[32] K. G. Paterson and V. Tarokh, "On the existence and construction of good codes with low peak-toaverage power ratios," IEEE Trans. Inform. Theory, vol. 46, pp. 1974-1987, Sept. 2000.

[33] K. Sathananthan and C. Tellambura, "Coding to reduce both PAR and PICR of an OFDM signal," IEEE Commun. Lett., vol. 6, pp. 316-318, Aug. 2002.

[34] X. Li and L. J. Cimini, Jr., "Effects of clipping and filtering on the performance of OFDM," IEEE Commun. Lett., vol. 2, pp. 131-133, May 1998.

[35] R. O'Neill and L. B. Lopes, "Envelope variations and spectral splatter in clipped multicarrier signals," in Proc. PIMRC'95, vol. 1, Toronto, Canada, Sept. 1995, pp. 71-75.

[36] J. Armstrong, "Peak-to-average power reduction for OFDM by repeated clipping and frequency domain filtering," Electronics Letters, vol. 38, pp. 246-247, Feb. 2002. 
[37] H.-G. Ryu, B.-I. Jin, and I.-B. Kim, "PAPR reduction using soft clipping and ACI rejection in OFDM system," IEEE Trans. Consumer Electron., vol. 48, pp. 17-22, Feb. 2002.

[38] H. Ochiai and H. Imai, "On clipping for peak power reduction of OFDM signals," in IEEE Global Telecommun. Conf., 2000. (GLOBECOM'00)., vol. 2, Nov. 27-Dec. 1, 2000, pp. 731-735.

[39] — , "Performance analysis of deliberately clipped OFDM signals," IEEE Trans. Commun., vol. 50, pp. 89-101, Jan. 2002.

[40] C. Berrou, A. Glavieux, and P. Thitimajshima, "Near shannon limit error-correcting coding and decoding: Turbo-codes," in Proc. IEEE International Conference on Communications (ICC'93), Geneva, Switzerland, May 1993, pp. 1064-1070.

[41] C. Berrou, "Turbo codes: some simple ideas for efficient communications," in Proc. Seventh International Workshop on Digital Signal Processing Techniques for Space Communications, Sesimbra, Portugal, Oct. 1-3, 2001.

[42] S. L. Goff, A. Glavieux, and C. Berrou, "Turbo-codes and high spectral efficiency modulation," in Proc. IEEE International Conference on Communications (ICC'94), New Orleans, May 1994, pp. 645-649.

[43] B. L. Floch, M. Alard, and C. Berrou, "Coded orthogonal frequency division multiplex," Proc. of the IEEE, vol. 83, pp. 982-996, June 1995.

[44] M. Benedix, J. Ertel, and A. Finger, "Turbo coding for an OFDM-based wireless LAN at $17 \mathrm{GHz}$," in Proc. 7th International OFDM-Workshop, Sept. 10-11, 2002.

[45] J. G. Proakis, Digital Communications, 3rd ed. New York, NY: McGraw-Hill, 1995.

[46] S. Haykin, Communication Systems, 3rd ed. New York, NY: Wiley, 1994.

[47] D. Kim and G. L. Stuber, "Clipping noise mitigation for OFDM by decision-aided reconstruction," IEEE Commun. Lett., vol. 3, pp. 4-6, Jan. 1999.

[48] J. Tellado, L. M. C. Hoo, and J. M. Cioffi, "Maximum-likelihood detection of nonlinearity distorted multicarrier symbols by iterative decoding," IEEE Trans. Commun., vol. 51, pp. 218-228, Feb. 2003.

[49] P. Banelli and S. Cacopardi, "Theoretical analysis and performance of OFDM signals in nonlinear AWGN channels," IEEE Trans. Commun., vol. 48, pp. 430-441, Mar. 2000.

[50] J.-J. van de Beek, O. Edfors, M. Sandell, S. K. Wilson, and P. O. Borjesson, "On channel estimation in OFDM systems,' in Proc. of the IEEE Vehicular Technology Conference, VTC '95, vol. 2, Jul. 1995, pp. 815-819. 


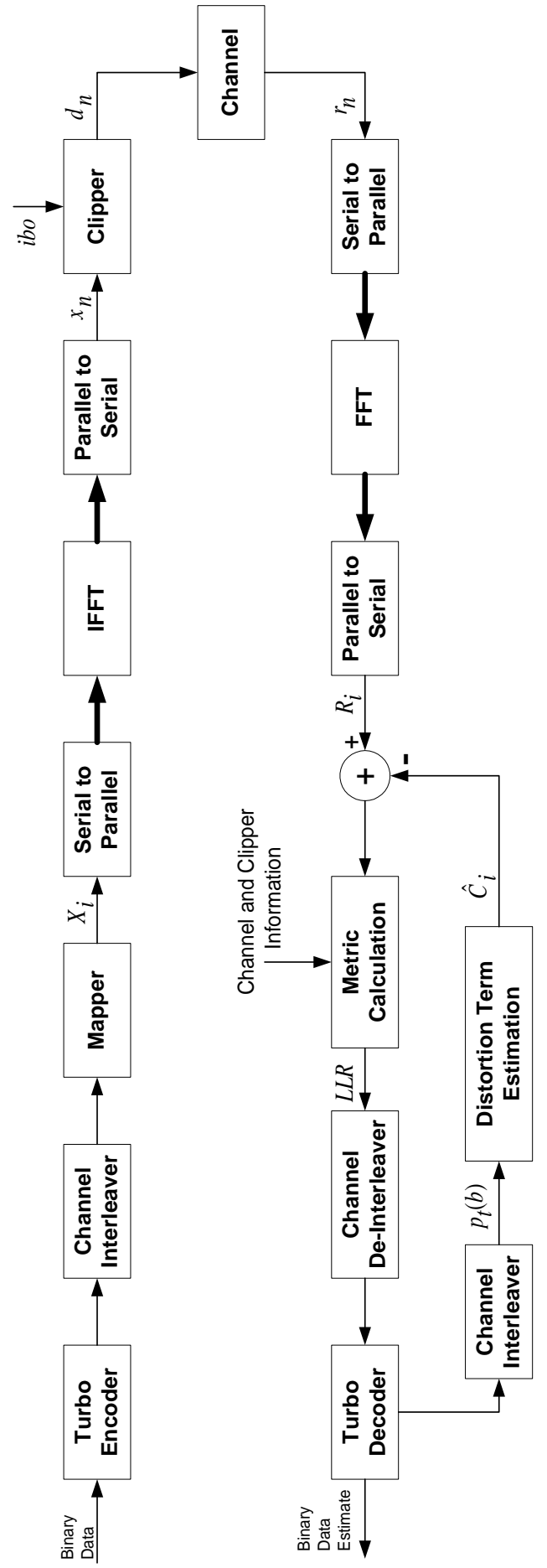

Fig. 1. The block diagram of the underlying OFDM transmission system. 


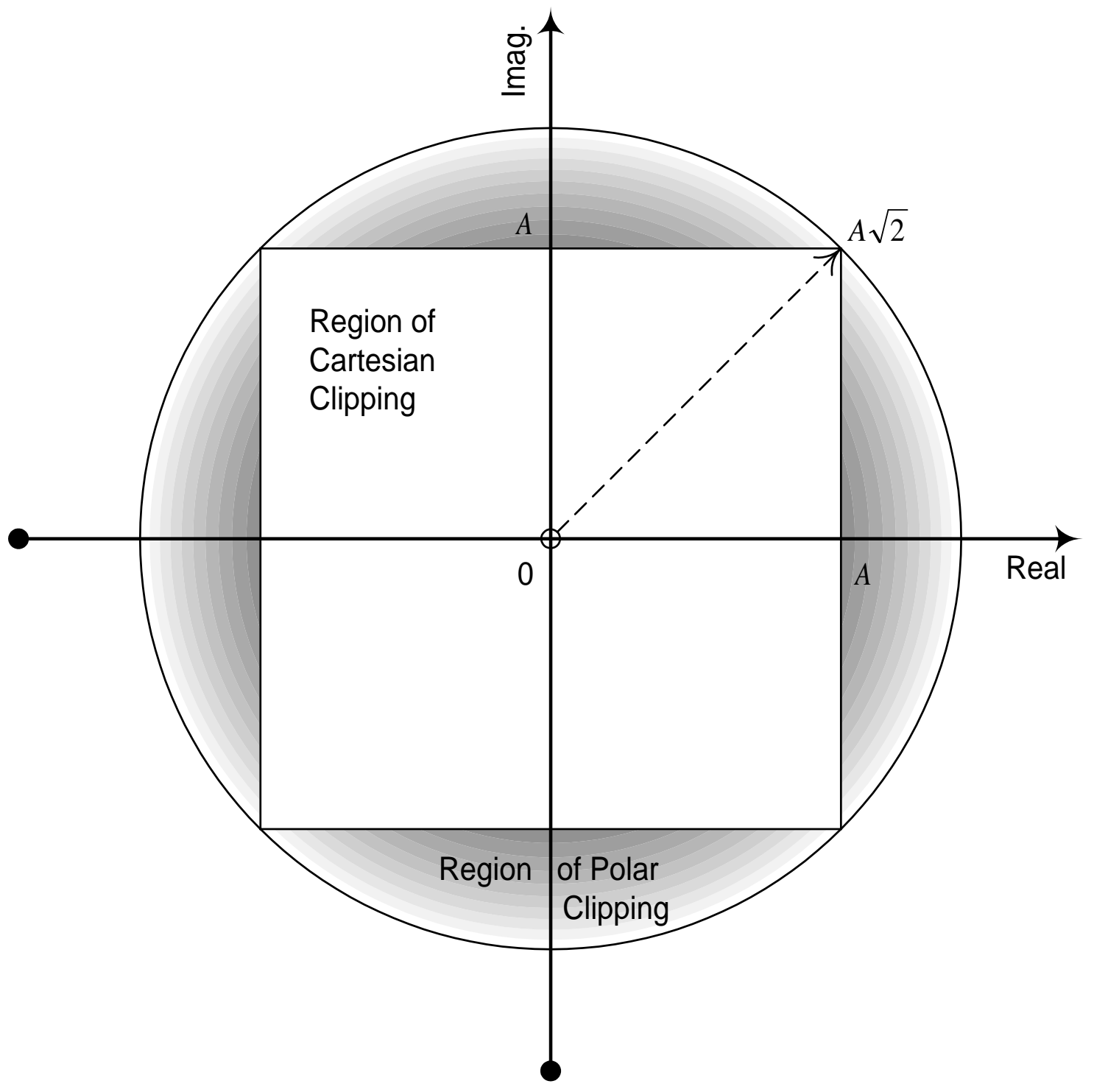

Fig. 2. A comparison of the polar and the Cartesian clipping. $i b o$ is the same for the both types and equal to $A^{2} / \sigma^{2}$. 


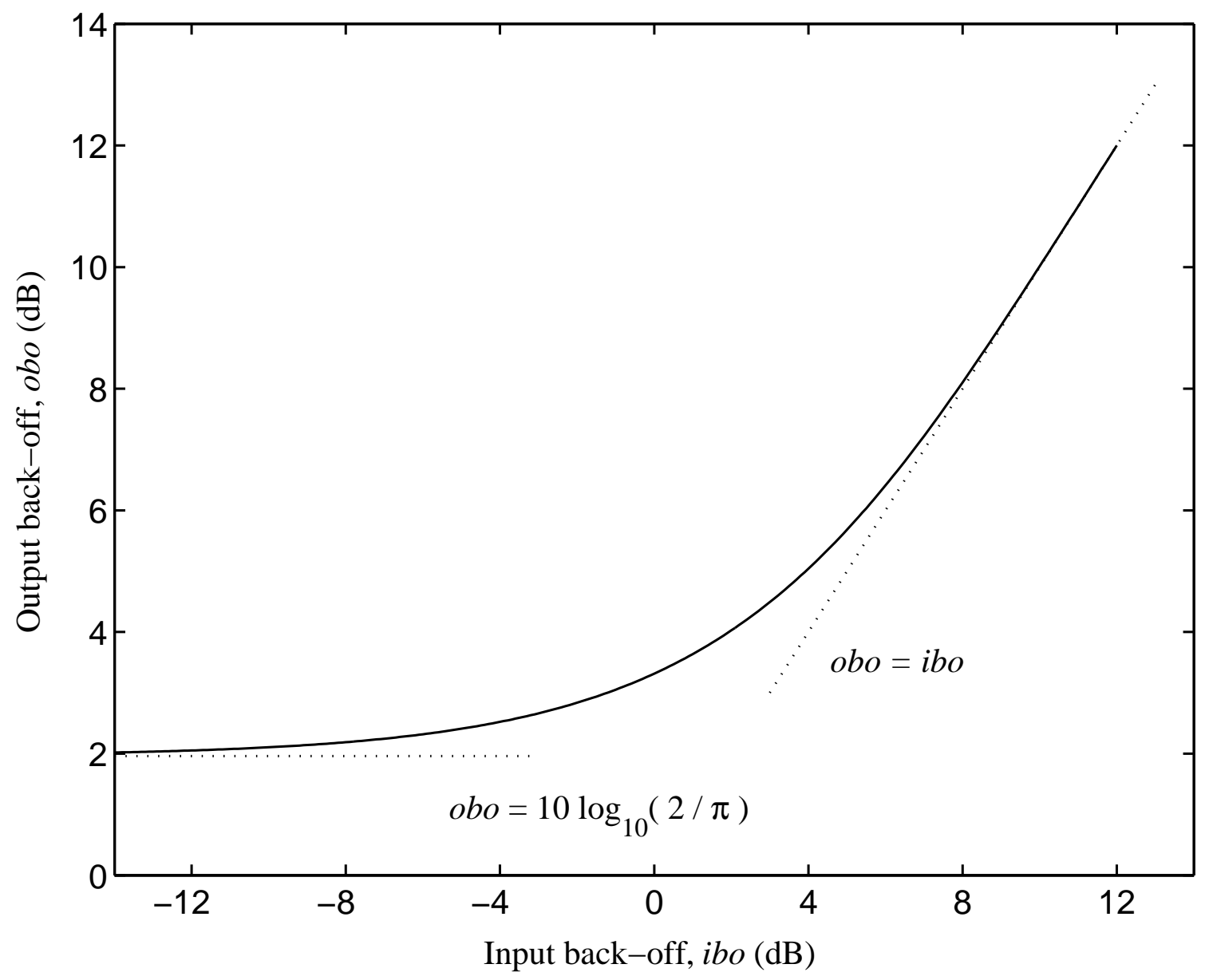

Fig. 3. obo, as a function of $i b o$. 


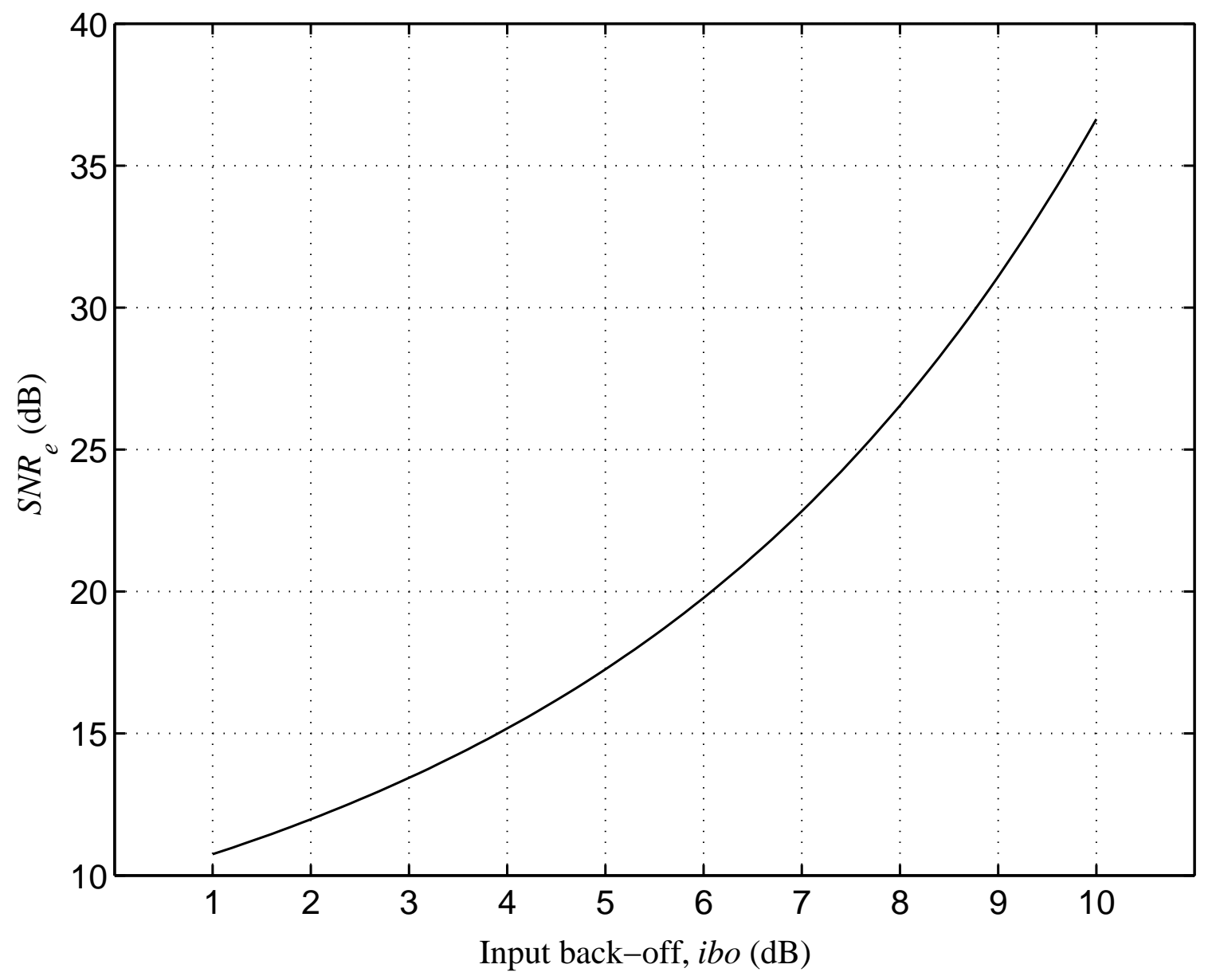

Fig. 4. The signal to nonlinear noise power ratio as a function of $i b o$ for the clipper. 


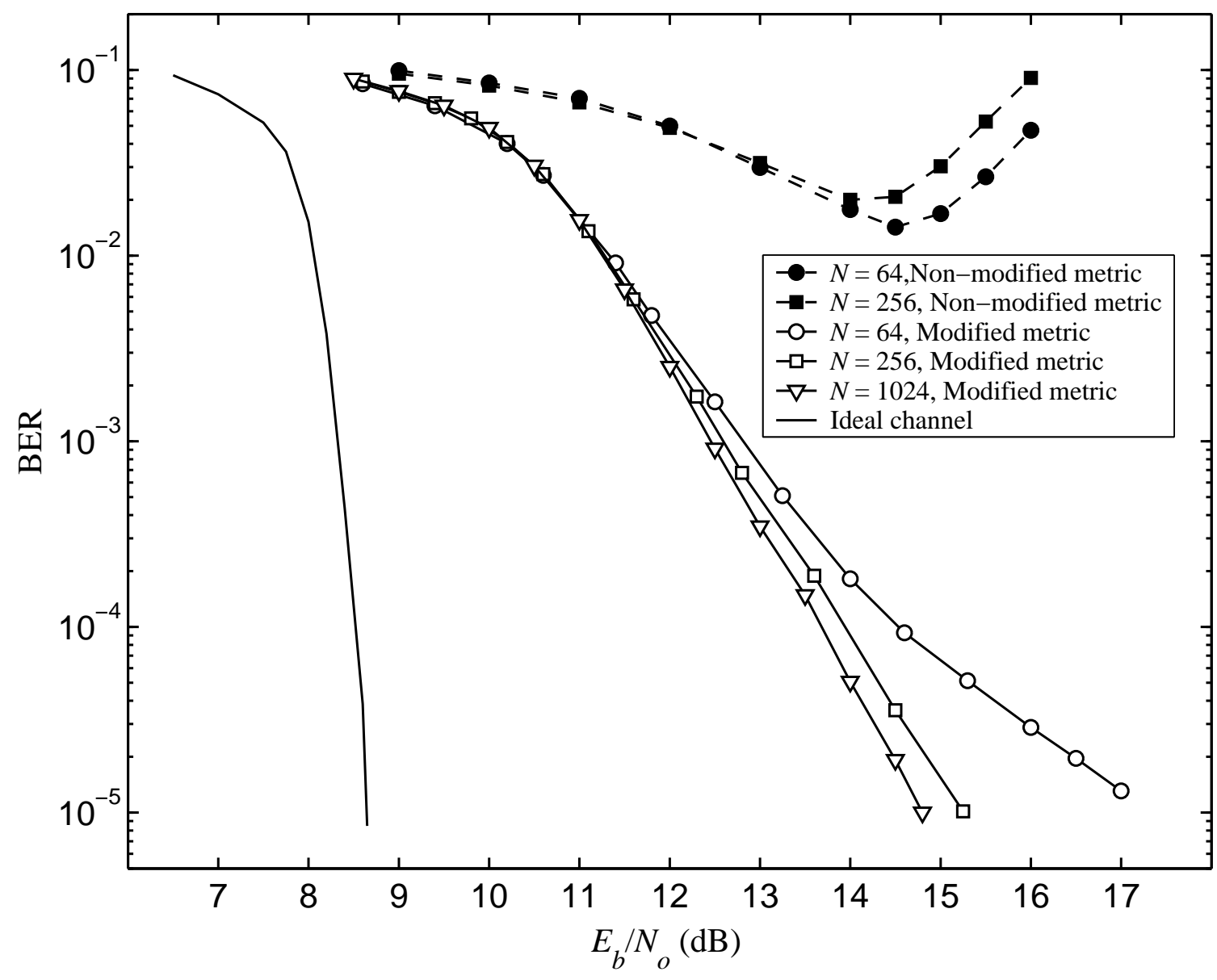

Fig. 5. The effect of the modified metric on 256-QAM OFDM systems with different subcarrier numbers. $i b o=5 \mathrm{~dB}$. 


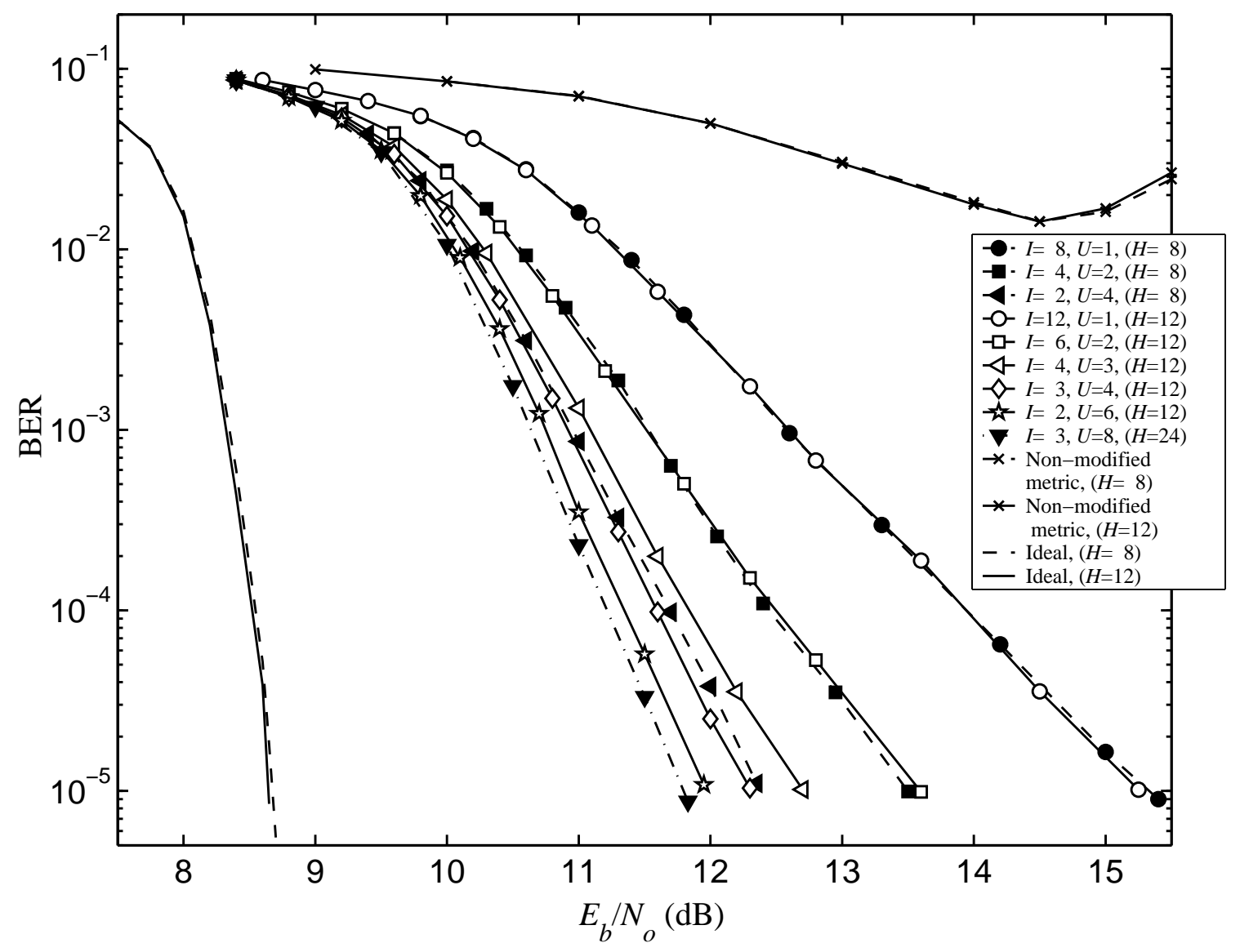

Fig. 6. The performance of the 256-subcarrier, 256-QAM OFDM system with the modified Turbo-decoder. $i b o=5 \mathrm{~dB}$. 


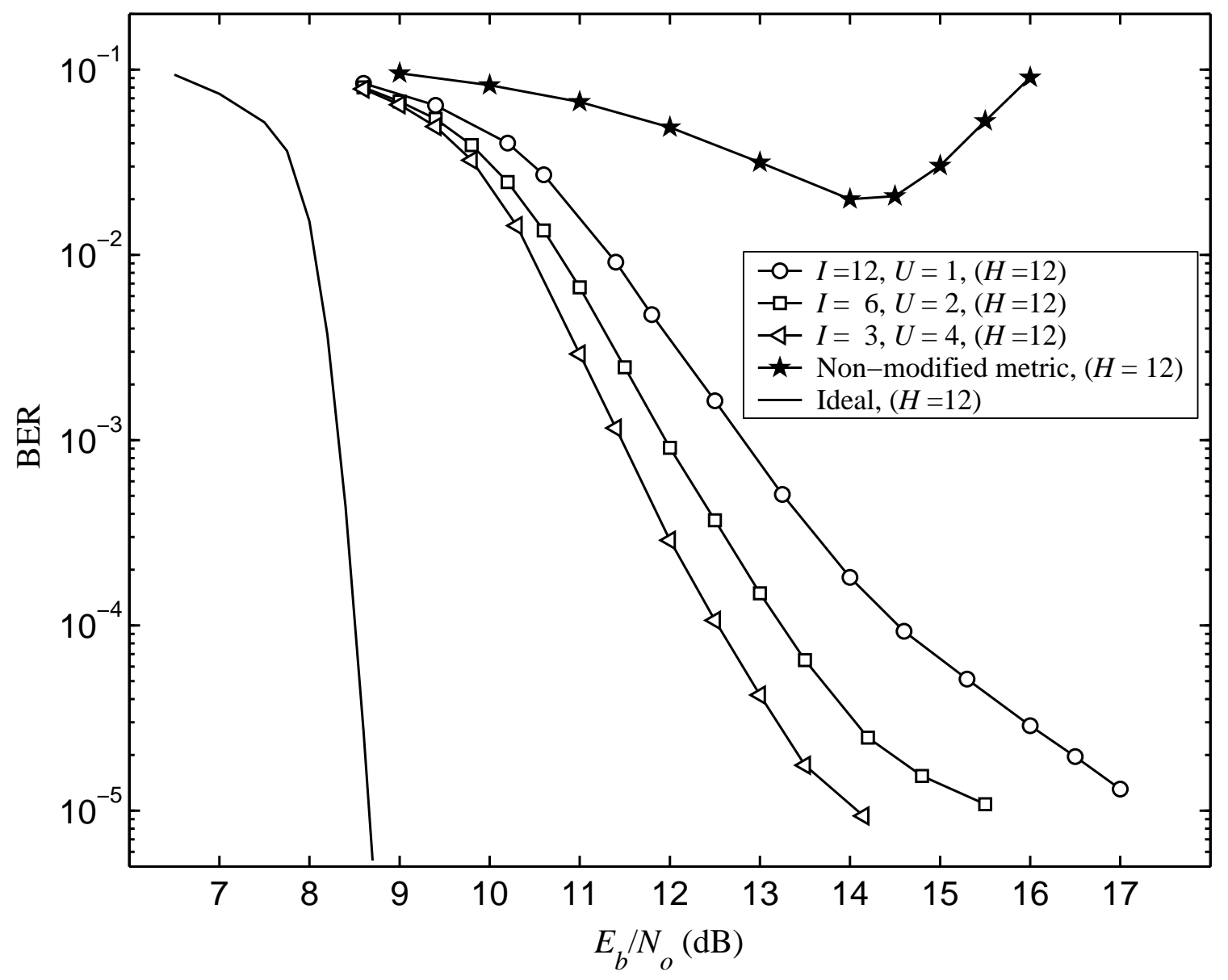

Fig. 7. The performance of the 64-subcarrier, 256-QAM OFDM system with the modified Turbo-decoder. $i b o=5 \mathrm{~dB}$. 


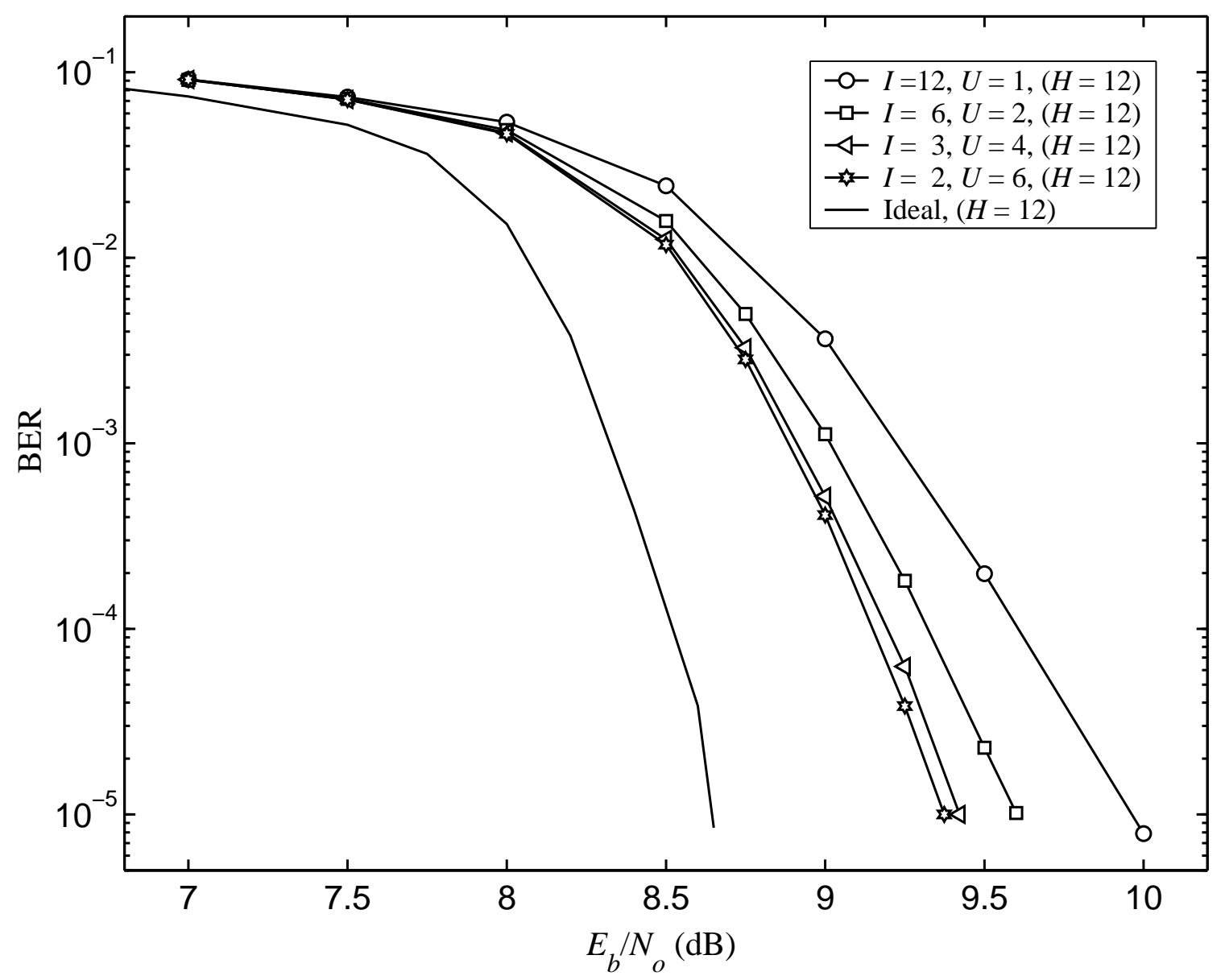

Fig. 8. The performance of the 256-subcarrier, 256-QAM OFDM system with the modified Turbo-decoder. $i b o=7 \mathrm{~dB}$. 


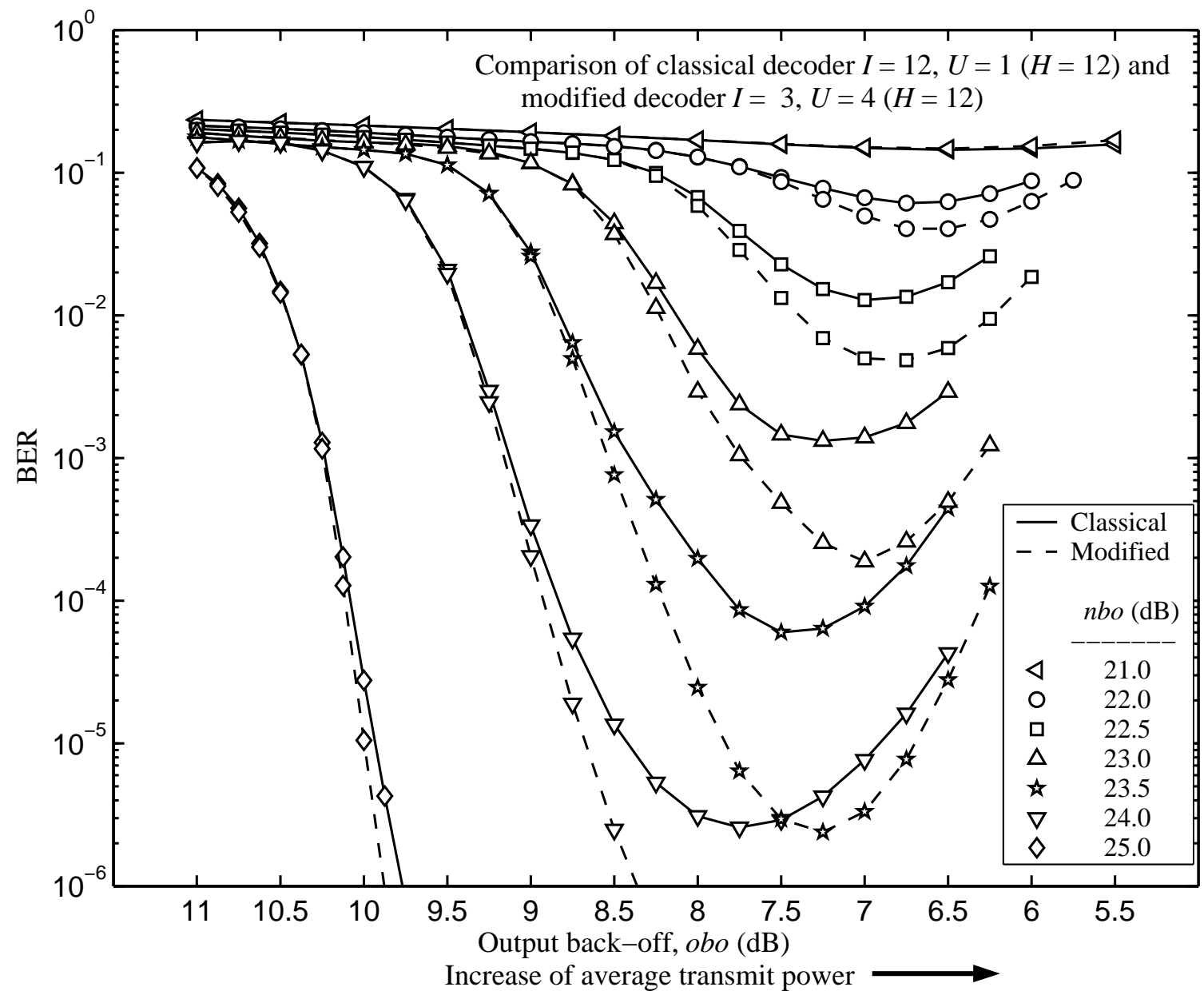

Fig. 9. A comparison of the modified, $I=3, U=4(H=12)$, and the classical, $I=12, U=1(H=12)$, Turbo-decoder in a practical situation. The results are for an 1024-subcarrier, 256-QAM OFDM system. 

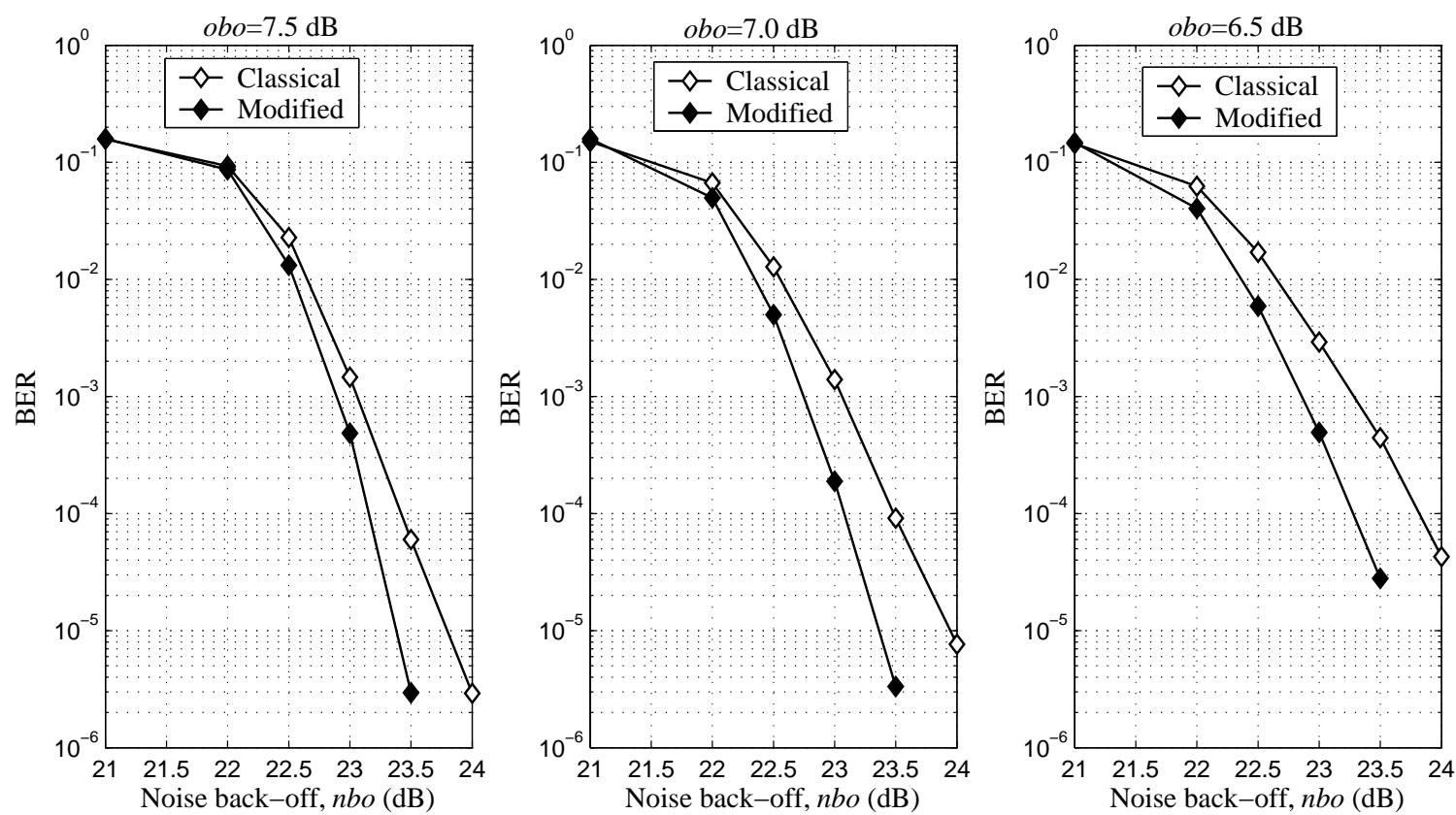

Fig. 10. The BER as a function of $n b o$ for $o b o=6.5,7.0,7.5 \mathrm{~dB}$. A comparison of the modified Turbo-decoder, $I=3, U=4(H=12)$, and the classical Turbo-decoder, $I=12, U=1(H=12)$. The results are for an 1024-subcarrier, 256-QAM OFDM system. 


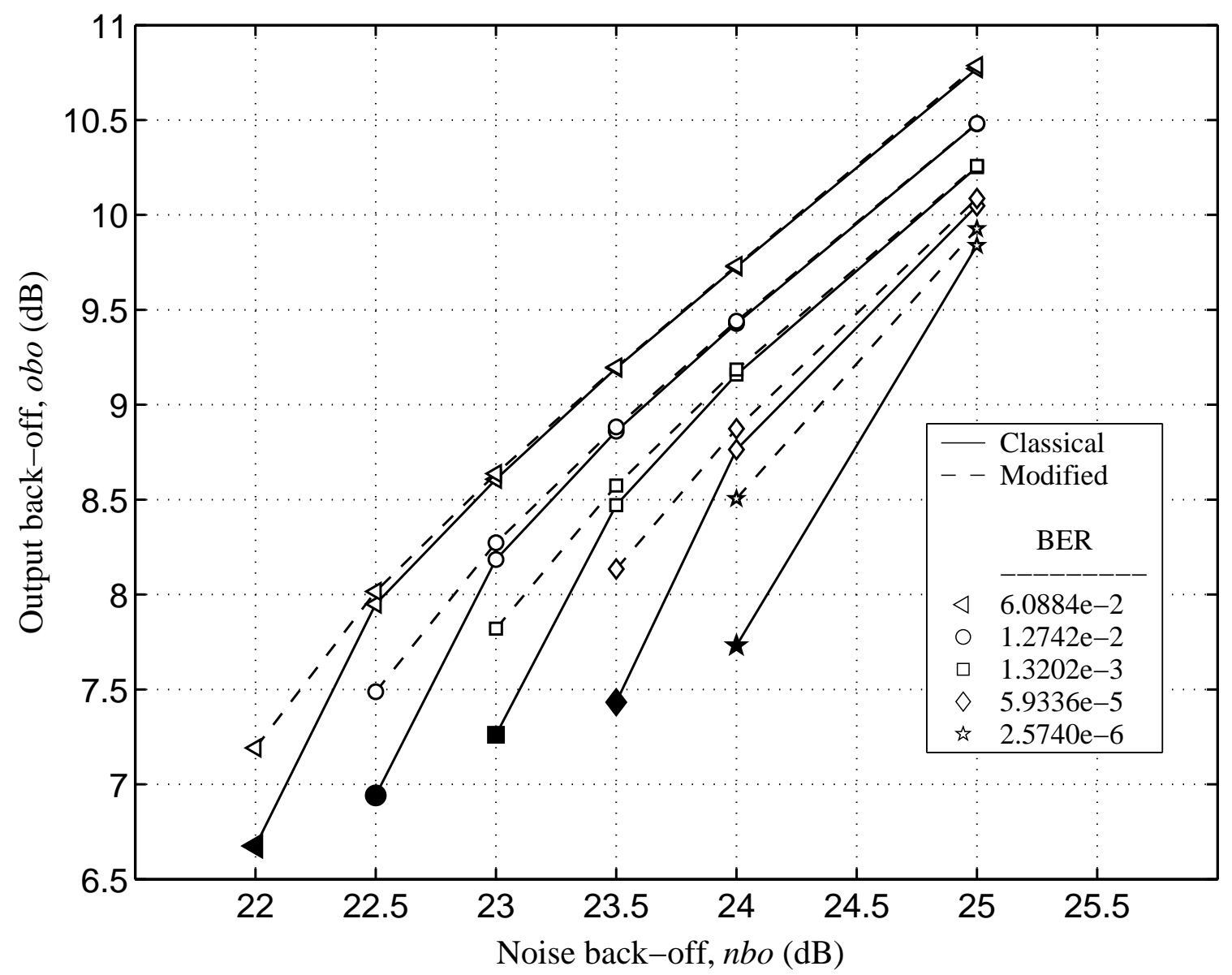

Fig. 11. $o b o$ as a function of $n b o$ for the several BERs. A comparison of the modified Turbo-decoder, $I=3, U=$ $4(H=12)$, and the classical Turbo-decoder, $I=12, U=1(H=12)$. The results are for an 1024-subcarrier, 256-QAM OFDM system. 


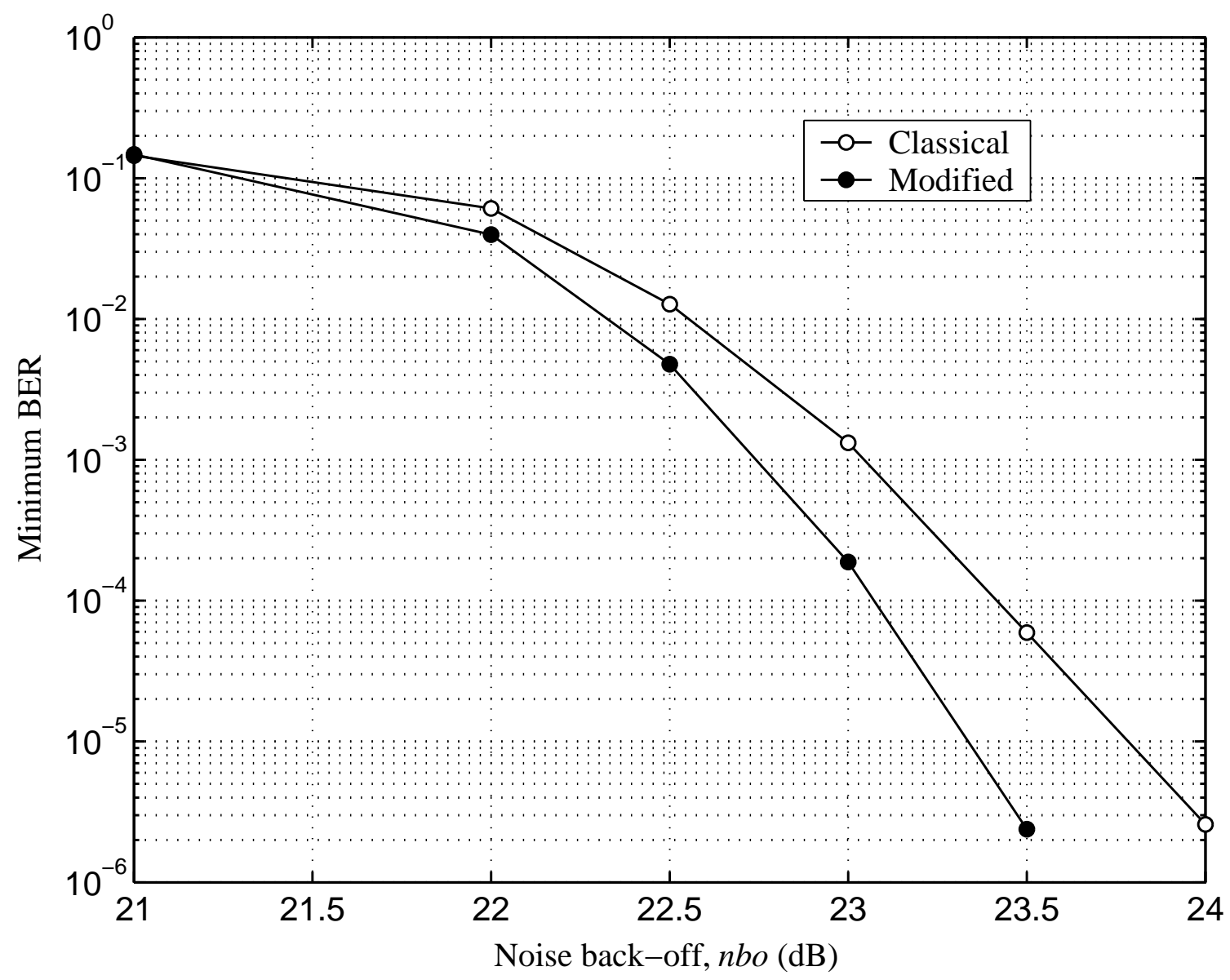

Fig. 12. A comparison of the modified Turbo-decoder, $I=3, U=4(H=12)$, and the classical Turbo-decoder, $I=12, U=1(H=12)$ at the optimum operating points. The results are for an 1024-subcarrier, 256-QAM OFDM system. (a) The minimum BER as a function of $n b o$. 


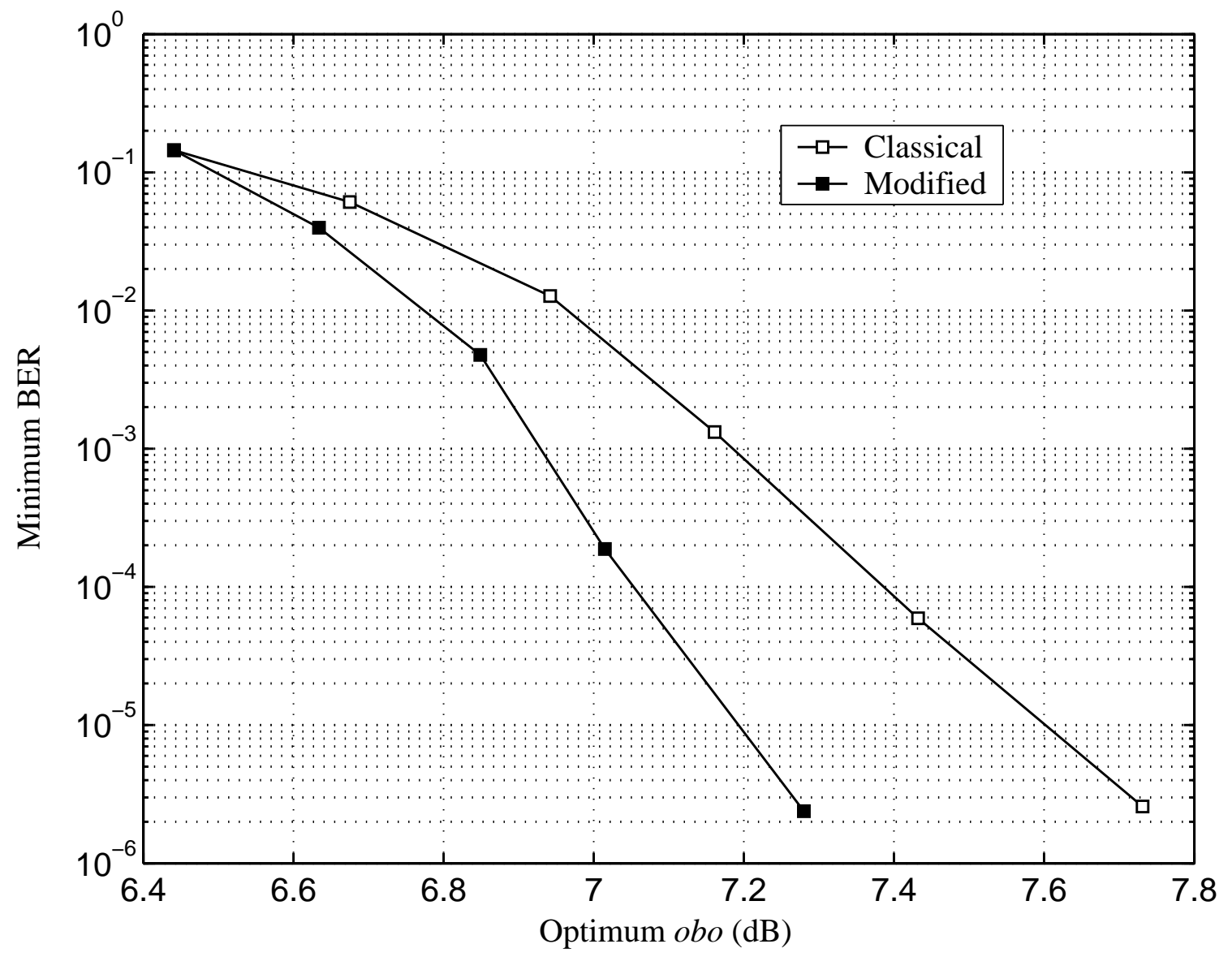

Fig. 12. (Continued.) (b) The minimum BER as a function of the optimum obo. 